\title{
Liste des thèses d'anthropologie soutenues en 2017
}

\section{Muséum national d'Histoire naturelle}

Christophe Coste : The costs of reproduction in evolutionary demography: An application of Multitrait Population Projection Matrix models

Goki Ly : Du système de parenté à la diversité génétique dans les populations humaines d'Asie du Sud-Est

Nina Marchi : A la croisée de l'anthropologie et de la biologie évolutive : diversité génétique et comportements migratoires en Asie Intérieure

Valentin Thouzeau: Inférer l'histoire des populations humaines à partir des diversités génétiques et linguistiques

\section{Université de Bordeaux}

Elsa Garot : Distinction entre processus pathologique de type MIH (molar incisor hypomineralisation) et processus taphonomique par différentes méthodes de micro-analyse de l'émail dentaire

\section{Université Paris 1 Panthéon-Sorbonne}

Marie Dejonghe : Les nécropoles phéniciennes de méditerranée occidentale. Architectures et pratiques funéraires

\section{Université Paris Nanterre}

Nathalie Del Socorro : Les rites funéraires dans le royaume des Téménides et ses environs à la période archaïque

\section{Université Paul Valery Montpellier 3}

Elsa Ciesielski : La pratique celtique des «têtes coupées » en France Méditerranéennes : l'exemple du site $d u$ Cailar (Gard) au III siècle avant notre ère. Approche archéothanatologique et traitements informatiques des données

\section{Université Toulouse III Paul Sabatier}

Barbara Da Costa : Caractérisation de modèles pouvant modifier la bio-énergétique mitochondriale: syndrome de Leigh et haplogroupe de l'ADNmt

Etienne Cavaignac: Etude de la variabilité en fonction du sexe, de l'âge et de l'origine géographique de l'extrémité distale du fémur

Pradiptajati Kusuma : A la recherche des ancêtres asiatiques des Malgaches en Indonésie

Fatima-Zohra Mokrane : L'angioscanner pos-mortem et ses diverses applications : de l'imagerie archéologique à l'étude de la population pédiatrique post-mortem

Nicolas Reina : Les contraintes de charge asymétriques chez l'Homme moderne. Étude microCT sur l'os trabéculaire

Frédéric Savall : Estimation de l'âge au décès d'individus français contemporains. Apport d'un échantillon virtuel à la méthode de Brooks et Suchey 